\title{
The NTP Experimental Platform for Heterogeneous Wireless Sensor Networks
}

\author{
Sheng-Po Kuo', Chun-yu Lin², Yueh-Feng Lee ${ }^{4}$, Hua-Wei Fang ${ }^{5}$, Y.-W. Peter Hong', \\ Hwa-Chun Lin ${ }^{2}$, Yu-Chee Tseng ${ }^{1}$, Chung-Ta King ${ }^{2}$, and Chin-Liang Wang ${ }^{*}{ }^{*}$ \\ 'Department of Computer Science, National Chiao Tung University, Taiwan \\ 2Department of Computer Science, National Tsing Hua University, Taiwan \\ ${ }_{3}^{3}$ Institute of Communications Engineering, National Tsing Hua University, Taiwan \\ ${ }^{4}$ Information \& Communications Research Labs., Industrial Technology Research Inst., Taiwan \\ ${ }^{5}$ Networks and Multimedia Institute, Institute for Information Industry, Taiwan
}

\begin{abstract}
This paper presents the NTP experimental platform for heterogeneous wireless sensor networks (WSN), which is built on the campus of National Tsing Hua University under the support of the National Science and Technology Program for Telecommunications (NTP) in Taiwan. The unique purpose of this testbed is to provide an open platform for research communities to test and to showcase their WSN systems and applications. The heterogeneous testbed is composed of three different sensor platforms: the III Zigbee Advanced Platform, the ITRI Sensor Platform and the Moteiv Tmote Platform. A middleware architecture is developed to support contextaware services (CASs) and location-based services (LBSs) across different sensor platforms. Specifically, a SENSORINFO gateway is implemented to hide the discrepancy of different sensor platforms from application developers, which allows application developers to deploy their CASs and/or LBSs while being oblivious to the underlying sensor hardware or system architecture. Moreover, to facilitate the experiments, an over-the-air (OTA) remote update function is available on the platform to enable reprogramming of sensors from a remote location. To demonstrate the unique features of this platform, we present two sample applications: the People Tracking System and the Green Building Monitoring System, which are examples of the LBS and the CAS, respectively.
\end{abstract}

\section{Categories and Subject Descriptors}

C.2.3 [Computer-Communication Networks]: Network Operations

\section{General Terms}

Experimentation

\footnotetext{
* The corresponding author of this paper is Chin-Liang Wang (e-mail: clwang@ee.nthu. edu.tw).

Permission to make digital or hard copies of all or part of this work for personal or classroom use is granted without fee provided that copies are not made or distributed for profit or commercial advantage and that copies bear this notice and the full citation on the first page. To copy otherwise, to republish, to post on servers or to redistribute to lists, requires prior specific permission and/or a fee.

TRIDENTCOM 2008, 17th- 20th Mar 2008, Innsbruck, Austria. Copyright () 2011- 2012 ICST ISBN 978-963-9799-24-0

DOI 10.4108/icst.tridentcom.2008.3115
}

\section{Keywords}

Context-aware services, location-based services, middleware architecture, over-the-air remote update, wireless sensor networks.

\section{INTRODUCTION}

Wireless sensor networks (WSNs) [1] typically consist of a large number of miniature devices that are able to take measurements from the physical environment, to process the retrieved data, to actuate appropriate responses and/or to provide the external world with desired information. When compared to traditional sensor networks, WSNs gain in the sense that it is easy to deploy, programmable, and dynamically reconfigurable. These features have led to many interesting applications in industrial control, household and community safety, medical care, ecological environment tracking, stock control, battlefield reconnaissance, and hazards mitigation, etc.

Following the research trend, both academic and industrial communities have actively promoted research in the field of WSNs. However, the main bottleneck encountered in these efforts is the lack of a large-scale experimental environment to test and to verify newly developed systems or ideas. In this paper, we present the architecture and design of a heterogeneous WSN platform recently deployed in Taiwan under the support of the National Science and Technology Program for Telecommunications (NTP). This project has been targeted at providing an open platform for academia, industry, and research institutes to perform WSN experiments and to showcase new WSN systems and applications.

The main features of the deployed platform are heterogeneity, interoperability, and openness. More specifically, the platform consists of three different sensor systems, namely, the Moteiv Tmote sensor platform [2], the ITRI sensor platform, and the III ZigBee Advanced Platform [3], and can easily incorporate sensor systems developed by other researchers as well. Different WSN systems are able to intercommunicate based on standardized protocols, such as Zigbee and IEEE 802.15.4, and to interoperate under common applications. More importantly, we open the platform to the public for researchers to develop their own upper-tier sensor applications as well as bottom-tier sensor systems. Over-the-air (OTA) remote update functionalities and web-based interfaces are provided for users to upload their applications or sensor images at ease [4].

Several sensor network testbeds have been deployed with similar functionalities. For example, Kansei [5] is a sensor network 

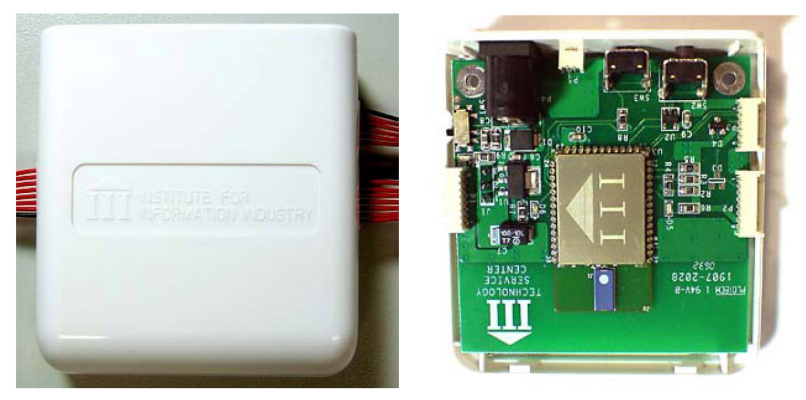

Figure 1: The top view of III ZigBee Device (Jennic platform).

testbed at Ohio State University that is heterogeneous in the sense that it is built on top of three types of sensor devices, namely, static, portable, and mobile sensor nodes. It also provides software components enabling the integrated experiments on these sensor devices to support complex experiments. NESTbed [6] is another testbed deployed at Clemson University that is used to facilitate the development of at-scale WSN applications. It is especially designed to support rapid, ad-hoc experimentations, in-situ debugging, and profiling in WSN applications.

In the proposed NTP experimental platform, we instead focus on the interoperability over different sensor hardware platforms. Standardized protocols, such as Zigbee and IEEE 802.15.4, are employed to guarantee intercommunication between the heterogeneous sensors. A middleware architecture is proposed along with various application programming interfaces (API) to facilitate the design of sensor network applications (namely, location-based services (LBSs) and context-aware services (CASs)) and to help shield application developers from the details of the implementation over heterogeneous platforms. Two sample applications are given to demonstrate the effectiveness of this testbed: the People Tracking System and the Green Building Monitoring System [see Section 4], which are examples of the LBS and the CAS, respectively.

The remainder of this paper is organized as follows. In Section 2, we introduce the hardware and software specifications of the experimental platform. The middleware architecture is presented in Section 3 and two pilot applications in Section 4. Finally, we conclude this paper in Section 5.

\section{SYSTEM PLATFORM}

Our WSN experimental platform is deployed on the campus of National Tsing Hua University (NTHU) in Taiwan. The indoor section is located in the NTHU library, and the outdoor section covers the square around the library and the nearby Cheng Kung Lake. Three different sensor network platforms, each with 50 sensor nodes, have been deployed on three different floors of the NTHU library. The platforms consist of the III ZigBee Advanced Platform, the ITRI Sensor Platform, and the Moteiv Tmote system, which are detailed below. The Tmote system also extends to the outdoor environment.

\subsection{Hardware Specifications}

\section{1) III ZigBee Advanced Platform (IZAP)}

The III ZigBee Advanced Platform (IZAP) consists of 50 III ZigBee Device (IZD), as shown in Fig. 1, that are manufactured and deployed by the Institute for Information Industry (III) in Taiwan. Each device consists of the Jennic JN5121 module with a chip antenna, 3 general I/O ports, 4 switches, a two-pin $3 \mathrm{~V}$ battery connector and a 5V DC jack, and provides the ZigBee/IEEE 802.15.4

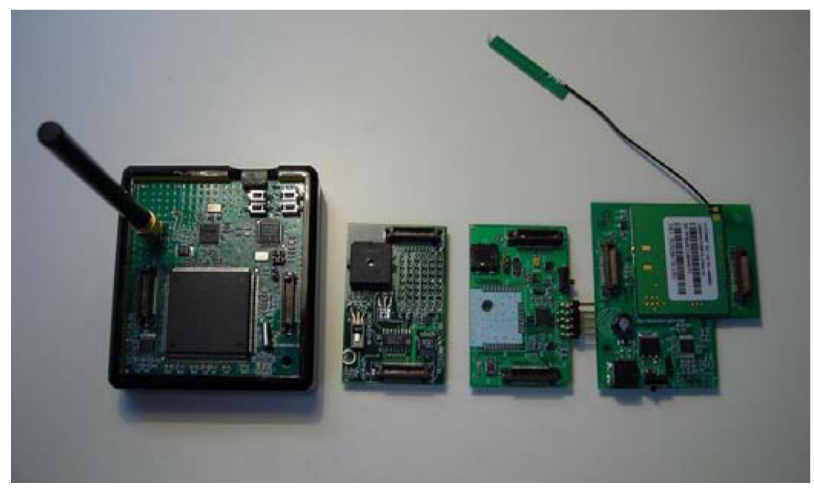

Figure 2: The ITRI 32-bit ZigBee sensor node and sensor boards.

standardized communication interfaces [7,8].

2) ITRI Sensor Platform

The ITRI sensor platform, developed by the Industrial Technology Research Institute (ITRI) in Taiwan, is also based on the ZigBee and IEEE 802.15.4 standard. Each sensor node is equipped with an ARM-based processor and a Chipcon CC2420 radio chip. The operating system is Linux 2.4 and the protocol stack of ZigBee and IEEE 802.15.4 is implemented as a kernel module. Each sensor node is equipped with a basic sensor board that contains four types of sensors: temperature, luminance, humidity, and sound, which can be easily replaced with sensor boards that have different sensing capabilities. The 32-bit ZigBee node and corresponding sensor boards are shown in Fig. 2.

3) Moteiv Tmote Sensor Platform

The Moteiv Tmote Sensor Platform features ultra low power, large storage, high data rate, and IEEE 802.15.4 compliant wireless sensor nodes that were developed by Moteiv [2]. Tmote Sky and Tmote Connect serves as the main components of this platform. Specifically, Tmote Sky is equipped with $10 \mathrm{~KB}$ RAM, $48 \mathrm{~KB}$ ROM, $1 \mathrm{MB}$ flash and operates under Tiny OS. It uses a 250kbps 2.4GHz IEEE 802.15.4 Chipcon wireless transceiver and a $8 \mathrm{MHz}$ Texas Instruments MSP430 microcontroller. Tmote Connect operates as a gateway appliance, which connects Tmote Sky sensors to a wired Ethernet local area network.

\subsection{Remote Update Functionalities}

To facilitate experiments, remote update functionalities are also implemented on the testbeds. A web-based interface is given for users to update sensor images or sensor applications onto each platform. The update of sensor images is achieved over-the-air (OTA) on the III ZigBee Advanced Platform and the ITRI Sensor Platform [4]. For the Tmote platform, Moteiv provides a web-based geographical user interface to administrate the Tmote Sky sensors remotely through Tmote Connect gateways. The remote update procedure is easily conducted by the flash reprogramming function of Tmote Connect over TCP/IP sockets over the wired connection between Tmote Connect and Tmote Sky sensors.

\subsection{Interoperability}

The experimental platform currently supports interoperability over two platforms: the III Zigbee Advanced Platform and the ITRI Sensor Network Platform. Based on the 802.15.4 and ZigBee standard, we allow the nodes developed by both parties to roam among each other's platform. Many issues must be overcome in order to achieve interoperability among the two platforms. First of all, both 


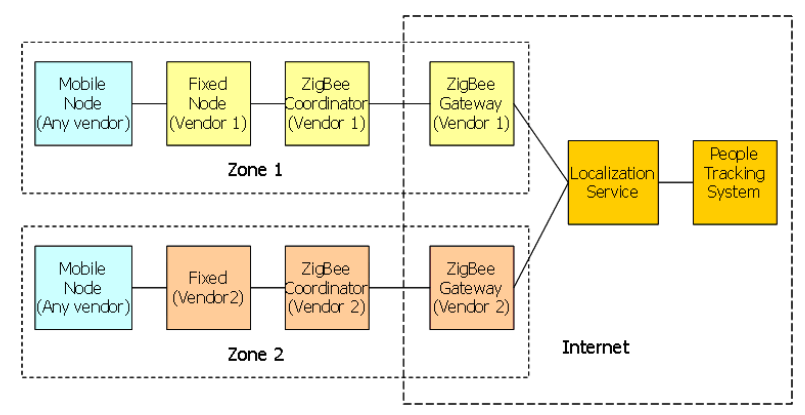

Figure 3: Interoperable system architecture of the People Tracking System.

the III and ITRI network components should follow an interoperable system architecture, as shown in Fig. 3. Second, on the ZigBee MAC/PHY level, the MAC addresses assigned to the nodes on one platform must not conflict with those on the other platform. Moreover, the nodes must be aware of the channel and the PAN ID used on the other platform so that it is able to successfully join the other network when needed. Third, on the application level, nodes from each platform must implement the same ZigBee profile, using the same cluster and endpoint, and generate the same type of application payload. Finally, each ZigBee gateway must transmit the same format of location data to the localization service subsystem.

The interoperability is tested with the people tracking system, where we learned that to achieve interoperability, the system requires a unified system architecture, well-defined system behavior, and intensive joint development and testing, which are all beyond the scope of the original protocol specifications. Network homogeneity is only a necessary requirement to achieve application-level interoperability, but in no way sufficient.

\section{MIDDLEWARE ARCHITECTURE}

While many sensor network testbeds focus on lower-level (or bottom-tier) sensor systems, one of the main goals of our testbed is to provide a platform for development of upper-tier sensing applications, in particular, the development of different location-based services (LBSs) and context-aware services (CASs). For this purpose, we propose a middleware architecture that helps shield the underlying hardware and software discrepancies of the different sensor network platforms from the application developers. This allows the users to port services from one platform to the other or to deploy a common application across different sensor platforms without rebuilding their services or modifying their message exchange protocols for each sensor platform.

The middleware architecture consists of three major components, as shown in Fig. 4. To simplify the description of the system, we will focus our discussions on the localization (or positioning) applications in heterogeneous WSNs. However, the proposed middleware architecture is not limited to such applications. Suppose that each user is equipped with a mobile device that is capable of interacting with the sensor network and the Internet (perhaps via separate wireless interfaces). Our goal is to unify the inquiry process of sensor data to simplify the deployment of CASs and LBSs on our heterogeneous platform. For this purpose, the SENSORINFO gateway is designed with three main features. First, the gateway accepts remote procedure calls using web services, which is referred to as the SENSORINFO API. Second, for each WSN platform, a proprietary protocol for inquiring a variety of sensor data is implemented. Third, it enables exchange of well formulated XML mes-

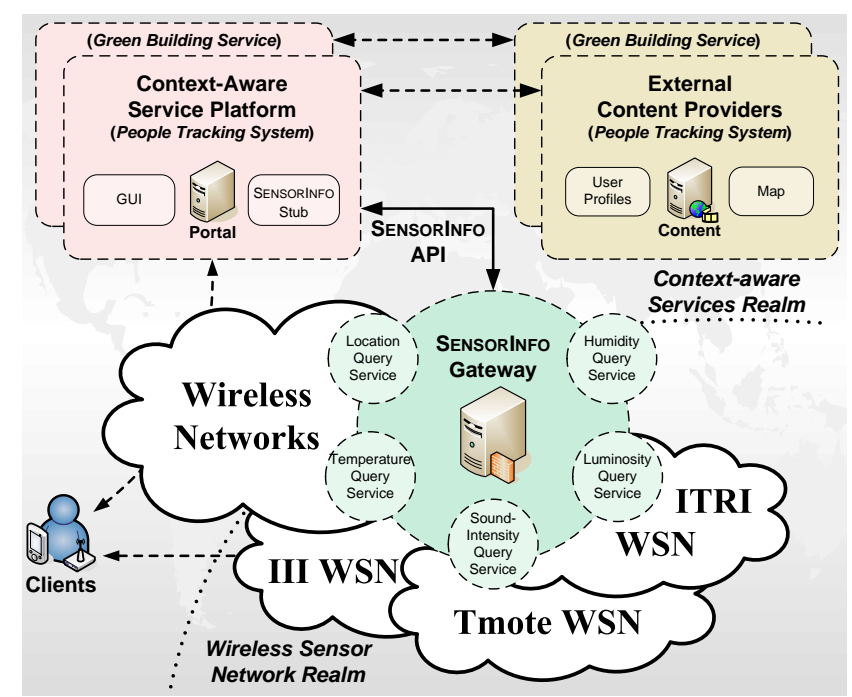

Figure 4: The proposed middleware architecture to support CASs and LBSs.

sages with different (location) servers to acquire sensing data (eg. users' current locations) from different sensor network platforms. The function of each component is explained as follows.

\subsection{SensorInfo Gateway}

To unify the inquiry process for different sensing data, we propose a gateway called SENSORINFO, which consists of two parts: the services and the collectors, as shown in Fig. 5. The services include Location Query Service, Temperature Query Service, Humidity Query Service, Luminosity Query Service, Sound Intensity Query Service, etc. Each service is able to accept a number of remote procedure calls in the form of web services and respond with unified data structures. For example, the Location Query Service accepts the getLocation() function call and returns the desired information through the LocationInfo data structure. In this case, the desired location information can be acquired from different sensor network platforms using the same service. On the other hand, the collectors are responsible for accessing, processing and buffering data from different sensor network platforms. Hence, they are typically platform-dependent. As examples, we will introduce below the Location Query Service and Temperature Query Service in more details.

The Location Query Service is designed according to two specifications: the Mobile Location Protocol (MLP) defined by the Open Mobile Alliance (OMA) [9] and the Terminal Location API [10], a Parlay X Web Service defined by the Parlay Group [11]. OMA [12] is an industry organization which targets at realizing usercentric mobile services across countries, operators, and mobile terminals. Its goal is to provide end-users with seamless mobile services. Among its many working groups, the Location Working Group, which continues most of the work originated by the former Location Interoperability Forum $(L I F)$, sets its goal on offering global LBSs on different networks and terminals. MLP is proposed for this purpose. MLP provides a simple yet secure message exchanging protocol for the Location Query Service to access the location collectors (refer to Section 3.2). All exchanged messages are defined by XML Document Type Definitions (DTD). Relying on the highly extensible property of XML, MLP is suitable to support cross-domain LBSs.

Although OMA MLP is a powerful message-exchange protocol, 


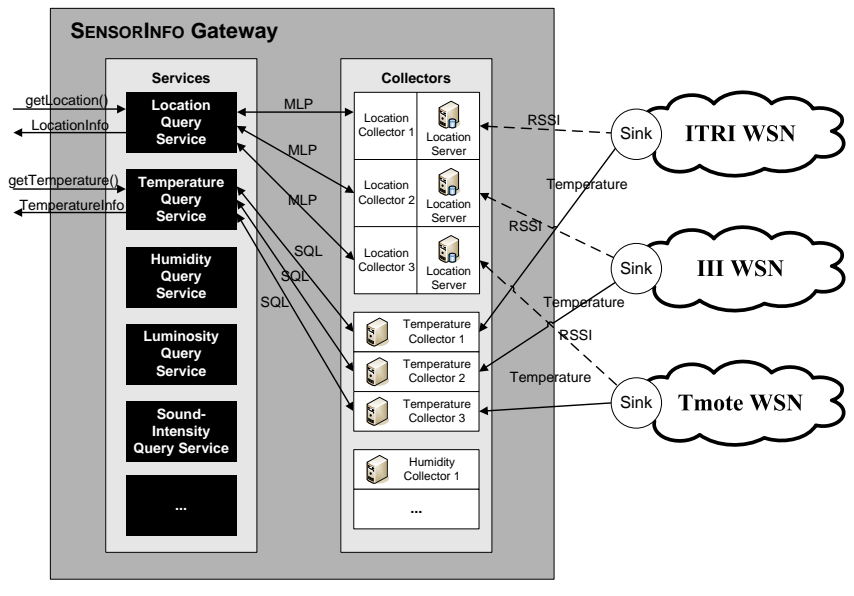

Figure 5: The design of the SENSORINFO gateway.

it is often inconvenient for application developers to compose requests and to interpret responses. Hence, a simple and general application programming interface is desired. The Parlay Group defines a series of powerful but simple web services for the telecommunication industry. For example, Terminal Location is one of these services that supports the inquiry of geographic information in terms of longitude, latitude, and altitude. Here, application developers can invoke the operation getLocation() to inquire the data and then interpret the information from the LocationInfo data structure, which contains the queried user's coordinate and the accuracy of this location information. The gateway exploits the advantages of web services and allows application developers to acquire users' locations without concerning specific programming languages.

The design of the Temperature Query Service is similar to the Location Query Service, except for the collector part. Since temperature information can be obtained directly from each sensor node without further processing, we can implement a polling mechanism in the collector to periodically acquire the last temperatures from sensor nodes. The polled data is stored in a database for further query. The Temperature Query Service has a set of web services which can access the database through the Structured Query Language (SQL). For example, application developers can invoke a getTemperature() function to inquire the database and encapsulate the result into a TemperatureInfo data structure, which contains the sensor node's address, type, temperature information, accuracy, and timestamp.

Similarly, we have also implemented other web services to acquire different types of sensor data, such as getHumidity() for the humidity estimations, getLuminosity() for the light intensity measurements, and getSoundIntensity() for the sound intensity of a specific sensor or a request area. When the SENSORINFO gateway receives a request, it will retrieve the corresponding sensor data from a WSN using a proprietary mechanism. Hence, application developers can deploy their CASs or LBSs easily without considering the details of the underlying sensor system.

Remark 1. SensorMap [16] is a Web-portal proposed by Microsoft Research with similar functionalities as the SENSORINFO gateway. Specifically, it provides tools and mechanisms for users to plug-in their sensors and publish the live sensing data over widearea sensor webs, and also provides a geo-centric web interface for users to perform geographical queries. However, the main focus of the SENSORINFO gateway is to provide a simple and unified way to access basic sensory data, such as location, temperature, hu- midity, luminosity, or sound intensity, across heterogeneous sensor network platforms. This allows application developers to deploy their CASs or LBSs easily without considering the details of the underlying sensor system, which is similar to the goal of the Open Service Architecture [11] in telecommunication systems.

\subsection{Location Servers}

To support LBSs, we adopt the pattern-matching techniques [13, 14] to provide the location estimates. In these systems, we first measure the signal strengths at predetermined target locations, which we call signal strength patterns, and record them in a location server. When the location of an object is desired, the received signal strength pattern of this object is measured and compared with those of the training locations. Advanced signal processing techniques can then be applied to interpolate the object's location. In contrast to this centralized scheme, decentralized positioning methods have also been studied [15]. In such systems, sensors can make local measurements of the signal emitted by the object and compute its location. The object's location is stored in a decentralized fashion in the network and can be inquired by the location server either periodically or on demand.

As discussed above, different localization schemes may require different ways to access the position information. In this case, location-based service providers may need to modify their application software for each individual positioning system. The middleware architecture that we propose allows us to hide the discrepancy of different localization systems from application developers. With this architecture, LBSs can be supported across different WSN platforms or different positioning systems.

\subsection{Context-Aware Service Platform}

The context-aware service platform is a portal, which accepts user requests for CASs or LBSs. Its main function is to provide graphical user interfaces and render the information obtained from external content providers to the users based on their surrounding context, such as their current locations and/or nearby temperatures. These sensor data are either stored locally or can be retrieved realtime through the SENSORINFO Stub, which connects to the SENSORINFO gateway. The GUI could be some web interfaces.

\subsection{External Content Providers}

This component provides typical contents for services that are not context-aware. For instance, a navigation service can automatically show a user's current location on a map. In this service, the owner of the map content can be regarded as an external content provider. Then a user's location can be rendered on the map. For an environmental monitoring application, the context-aware service platform can retrieve the context observed by sensors and store them in a database owned by an external content provider. The data can be accumulated for future environmental analysis.

\subsection{Clients}

In this system, the users are equipped with mobile devices which are able to display the GUI of various applications, communicate with the context-aware service platform through a WiFi NIC. They also wear a portable badge to receive signal strengths from wireless sensors. The badge can be interoperable over the III platform and the ITRI platform because both of them are based on the 802.15.4 and ZigBee standard. For the pattern-matching localization technique [13], the observed signal strength samples of nearby sensor nodes are sent to the WSN location server by the users' badges. For the decentralized positioning methods [15], the signal-strength measurements and the estimated locations are obtained and recorded at the sensor nodes. 


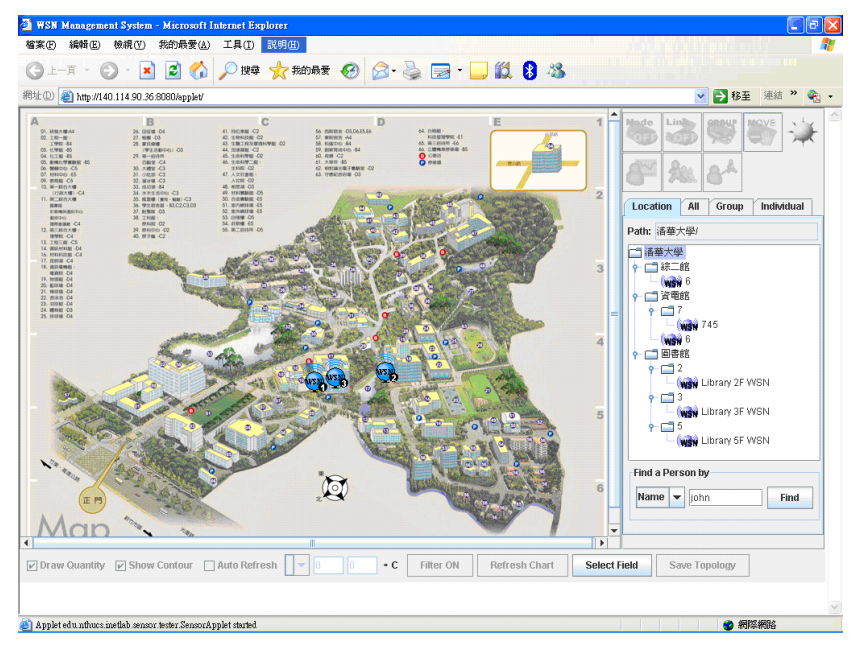

Figure 6: Main page of the People Tracking System.

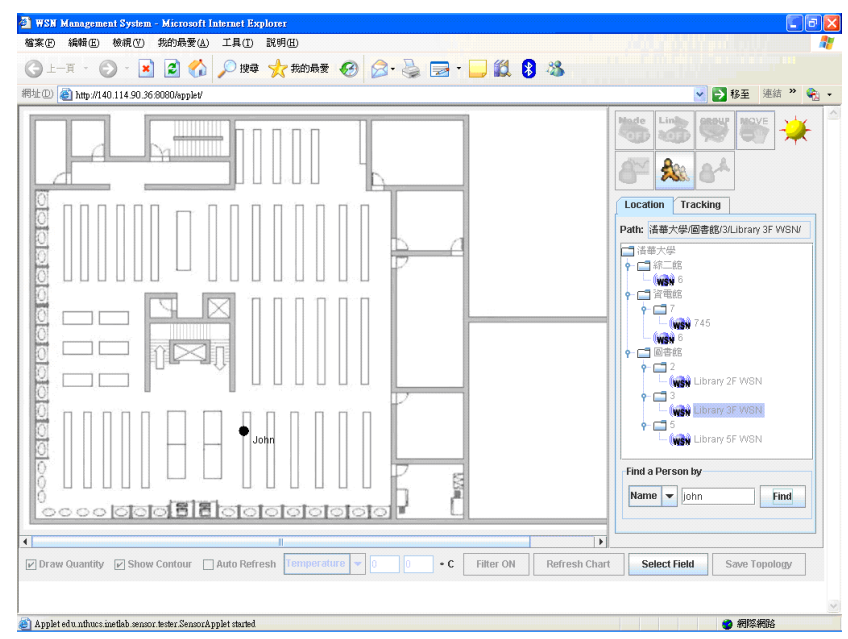

Figure 7: Search result of the People Tracking System.

\section{SAMPLE APPLICATIONS}

To demonstrate the proposed middleware architecture and to test the functionalities of the heterogeneous platform, we present the People Tracking System and the Green Building Monitoring System as two pilot applications. Both of them rely on the open web services provided by the SENSORINFO gateway. The discrepancy between the different sensor network platforms will be covered by the proprietary implementation between the SENSORINFO gateway and each WSN. Hence, these applications can be applied to different WSNs without additional modifications.

\subsection{People Tracking System}

The People Tracking system is a web-based application that is able to locate people (equipped with badges) within the sensor coverage area, as shown in Fig. 6. Users of this system are able to locate people or objects by entering sensor IDs or target names into the program. When the target (i.e. the person of interest) is found, the web page is directed to the map where the target is currently located, as shown in Fig. 7. The system provides two display modes: the non-tracking mode and the tracking mode. In the non-tracking mode, only the current location of the target is shown and is updated periodically. In the tracking mode, the web page displays a

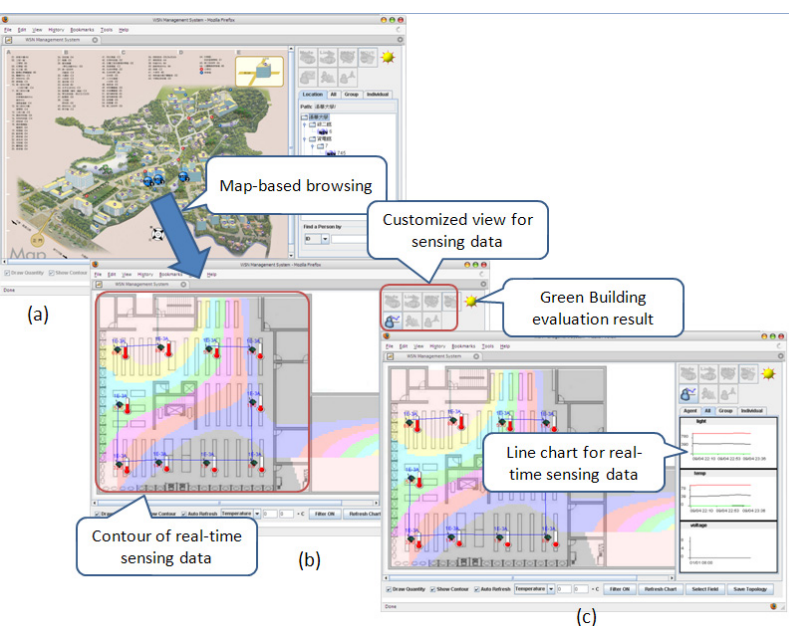

Figure 8: (a) The map interface, (b) the contour view, and (c) the line chart view of the implemented Green Building application.

track of the target by interpolating over its past location history. In addition, the system also allows users to browse over the people within certain areas.

The people tracking system consists of two types of sensor nodes: fixed nodes and mobile nodes. The fixed nodes are used as reference nodes in the localization service subsystem, which is the front-end of the people tracking system. The mobile nodes are portable nodes, e.g. badges, that are worn by people on campus. Fixed nodes from different sensor platforms are deployed around campus, each covering a different area. The people tracking system is interoperable over the III Zigbee Advanced Platform and the ITRI Sensor Network Platform, meaning that a badge from either platform may roam across the other platforms with no obstruction.

SENSORINFO gateway abstracts the inquiry of location by providing a unified interface without the technical detail of building a location severs. Two major benefits can be identified for people tracking system. Firstly, due to the interoperability, the tracked target can freely roam among different sensor platforms without becoming out-of-track. The API provided by the SENSORINFO gateway allows us to focus on the application logics while developing the people tracking system, such as tracking mode switching or web pages displaying. Secondly, although a pattern-matching based localization system is currently deployed, different localization algorithms can also be employed simply by replacing the location server in the SENSORINFO gateway, without affecting the people tracking system. This allows us to evaluate different sensor localization systems under the same application.

\subsection{Green Building Monitoring System}

In this application, we demonstrate how WSNs can help to better realize the concept of Green Buildings. Green Building is a practice to reduce the impact of buildings on the environment and human health. Restrictions and criterions on building materials, space design, and many other indirect environmental factors are used as guidelines to certificate a Green Building. The indirect evaluation tools are used because the actual Green Building standards require long-term monitoring, which is extremely costly and is laborintensive. Interestingly, WSN provides the technology needed to perform such environmental monitoring without human intervention. 
Following this concept, the Green Building Evaluator (GBE) is developed using a web-based graphical interface, as shown in Fig. 8. GBE evaluates the indoor environment of NTHU library using the rating system given in [17] , presenting the real-time evaluation results in several comprehensive ways, like contour, line chart, icons. These visualized presentations helps library manager better know about the indoor environment, and furthermore, based on the output of GBE, actuation can be performed adaptively to improve the comfort level within the environment, such as adjusting the airconditioning or the lighting.

The environmental data is retrieved by the GBE using the web services provided by the SENSORINFO gateway. GBE benefits from the infrastructure in following aspects. Firstly, sensor readings can be gathered without considering heterogeneity of sensor platforms. For example, GBE evaluates the sound-proof capability of the window by invoking getSoundIntensity() for the indoor/outdoor sound intensities and comparing them, while indoor reading is from one sensor platform and outdoor reading is from another. Moreover, with predefined interfaces and open standards, developers can only focus on the application related information, such as location or the sensing rate, rather than types of sensor platforms. Therefore, SENSORINFO gateway facilitates the development of such applications.

\section{CONCLUSIONS}

In this paper, we have presented the recently deployed NTP experimental platform for heterogeneous WSNs. The platform has been aimed to provide an open platform for research communities in Taiwan to test and to showcase their WSN systems and applications. This heterogeneous platform is composed of three different sensor platforms: the III Zigbee Advanced Platform, the ITRI Sensor Platform and the Moteiv Tmote Platform. To facilitate the application development over the heterogeneous sensor environment, a middleware system, i.e. the SENSORINFO gateway, has been implemented to hide the underlying hardware discrepancy from application developers. Many web-service based API have been realized on the gateway to facilitate the development of various contextaware or location-based applications over heterogeneous platforms. Finally, two pilot applications have been implemented to demonstrate the unique features of this heterogeneous WSN platform, i.e., the People Tracking System and the Green Building Monitoring System.

\section{ACKNOWLEDGEMENT}

This work was supported by the National Science Council of the Republic of China under Grants NSC 94-2219-E-007-009 and NSC 95-2219-E-007-008.

\section{REFERENCES}

[1] I. Akyildiz, W. Su, Y. Sankarasubramanian, and E. Cayirci. A survey on sensor networks. IEEE Communications Magazine, 40(8):102-114, August 2002.
[2] Moteiv Website - http: / /www.moteiv.com/.

[3] III ZigBee Advanced Platform Website http://zigbee.iii.org.tw/.

[4] Y.-F. Lee and C.-C. Shen. An transaction-based approach to over-the-air programming in wireless sensor networks. In Proceedings of IEEE International Symposium on Communications and Information Technologies (ISCIT), 2007.

[5] E. Ertin, A. Arora, R. Ramnath, M. Nesterenko, V. Naik, S. Bapat, V. Kulathumani, M. Sridharan, H. Zhang, and H. Cao. Kansei: A testbed for sensing at scale. In Proceedings of International Conference on Information Processing in Sensor Networks (IPSN), pp. 399-406, 2006.

[6] A. R. Dalton and J. O. Hallstrom. A file system abstraction and shell interface for a wireless network testbed. In Proceedings of International Conference on Testbeds and Research Infrastructures for the Development of Networks \& Communities (TridentCom), May 22, 2007.

[7] Zigbee Alliance - http: / / www . zigbee.org/.

[8] IEEE standard for information technology telecommunications and information exchange between systems - local and metropolitan area networks specific requirements part 15.4: wireless medium access control (MAC) and physical layer (PHY) specifications for low-rate wireless personal area networks (LR-WPANs), 2003.

[9] Mobile Location Protocol v3.1 -

http: / /www. openmobilealliance.org/

release \_program/docs/MLP/

OMA-LIF-MLP-V3\_1-20040316-C.pdf.

[10] Terminal Location API http: / / www . parlay.org / imwp/idms / popups / pop \_download.asp?ContentID=11418.

[11] The Parlay Group http: / /www.parlay.org/en/index.asp.

[12] Open Mobile Alliance http: / / www . openmobilealliance.org.

[13] P. Bahl and V. N. Padmanabhan. RADAR: An in-building RF-based user location and tracking system. In Proceedings of IEEE INFOCOM, volume 2, pages 775-784, 2000.

[14] S.-P. Kuo, B.-J. Wu, W.-C. Peng, and Y.-C. Tseng. A cluster-enhanced localization framework in wireless networks. In Proceedings of IEEE International Conference on Mobile Ad-hoc and Sensor Systems (MASS), 2007.

[15] C.-L. Wang, Y.-W. Hong, and Y.-S. Dai. A decentralized positioning method for wireless sensor networks based on weighted interpolation. In Proceedings of IEEE International Conference on Communications (ICC), 2007.

[16] S. Nath, J. Liu, and F. Zhao. SensorMap for wide-area sensor web. Computer, 40(7):90-93, 2007.

[17] C.-M. Chiang and C.-M. Lai. A study on the comprehensive indicator of indoor environment assessment for occupants' health in Taiwan. Building and Environment, 37(4): 387-392, 2002. 